\title{
Использование вторичных мишеней в энергодисперсионных рентгенофлуоресцентных спектрометрах Genius IF
}

\author{
А. Е. Каменщиков, И. В. Лавренов, В.Е.Фомин \\ ООО «НКЦ «ЛАБТЕСТ», МосКва \\ alexkam@lab-test.ru
}

УДК 543.427.4, 543.07

Статья поступила в редакцию 3.09.2019

Статья принята к публикации 25.09.2019

\begin{abstract}
Рентгенофлуоресцентный анализ (РФА) - один из лучших аналитических методов элементного анализа самых различных проб: твердых веществ, жидкостей и порошков. Описан принцип работы аппаратуры для энергодисперсионного (ЭД) РФА. Обсуждаются способы увеличения чувствительности путем улучшения эффективности регистрации сигнала рентгеновского излучения с помощью применения фильтров и вторичных мишеней. Проведены эксперименты при прямом возбуждении образца, а также с применением фильтров и вторичных мишеней. Продемонстрированы примеры практического применения, в которых наблюдается значительное увеличение соотношения сигнал / шум при использовании вторичных мишеней во всем спектральном диапазоне при соответствующем выборе материала мишени. Экспериментально показано влияние материала мишени на интенсивность линий аналитов.
\end{abstract}

\section{ВВЕДЕНИЕ}

Энергодисперсионный рентгенофлуоресцентный анализ (ЭД-РФА) является быстрым, достаточно точным и во многих случаях неразрушающим методом многоэлементного спектрального анализа образцов самой различной природы. Исследуемый материал может быть представлен в виде порошка, спрессованного либо сплавленного диска, жидкости или твердого образца, в целом ряде применений пробоподготовка минимальна. Рентгенофлуоресцентные спектрометры нашли применение в различных областях: экология и охрана окружающей среды (определение тяжелых металлов в почвах, осадках, воде и др.), геология и минералогия (качественный и количественный анализ почв, минералов, горных пород и др.), металлургия и химическая промышленность (контроль качества сырья, производственного процесса и готовой продукции), ювелирное производство (определение содержания ценных металлов), нефтяная индустрия (определение загрязнений нефти и топлива), пищевая промышленность (определение токсичных металлов в пищевых ингредиентах), сельское хозяйство (анализ микроэлементов в почвах и сельскохозяйственных продуктах), археология (определение элементного состава), искусство (экспертиза). эД-РФА также широко используется при проведении различных научных исследований.
Методом ЭД-РФА можно определять концентрации элементов от долей pрт в диапазоне атомных масс от $\mathrm{Na}(11)$ до U (92), а применение сверхтонких пленок для окошка детектора расширяет диапазон в сторону более легких элементов, вплоть до $\mathrm{F}(9)$. Классический способ добиться увеличения чувствительности при анализе конкретных элементов в тех или иных матрицах состоит в использовании фильтров, изготовленных из различных, заранее подобранных материалов. Однако этого может быть недостаточно для выделения узкой области возбуждения с целью определения наиболее проблематичных элементов в исследуемом образце. Решить возникающие проблемы при анализе различных типов образцов можно с помощью вторичных мишеней. Работу по исследованию свойств и возможностей вторичных мишеней проводили инженеры ООО «НКЦ «ЛАБТЕСТ» в демонстрационной лаборатории компании на спектрометре Genius IF производства Xenemetrix (Израиль) [1].

\section{ПРИНЦИП РАБОТЫ МЕТОДА}

Сегодня в РФА-спектрометрах чаще всего в качестве источников излучения используются рентгеновские трубки (рис. 1). Взаимодействуя с веществом образца, рентгеновское излучение частично поглощается и частично рассеивается. 


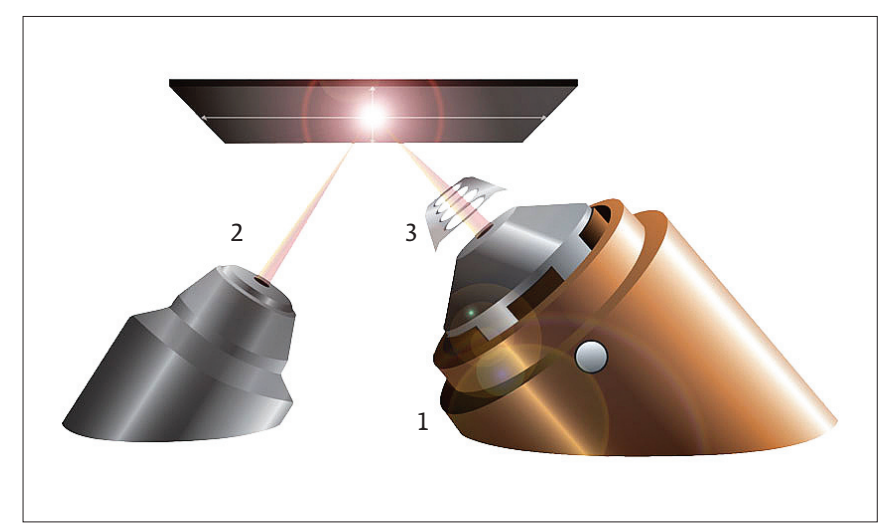

Puс. 1. Принцип работы метода: 1 - рентгеновская трубка; 2- ЭД-детектор; 3 - набор фильтров

Поглощенный фотон выбивает из внутренней электронной оболочки атома электрон, а на освободившееся место переходит электрон с более высокого энергетического уровня, испуская фотон характеристического излучения, свойственного конкретному химическому элементу (рис. 2). Кванты высокоэнергетического излучения, попадая на энергодисперсионный детектор (см. рис. 1), вызывают в нем пропорциональные их энергиям короткие импульсы напряжения. Эти импульсы затем оцифровываются и суммируются в спектр с помощью многоканального анализатора. В технологии ЭД-РФА не применяются прецизионные оптические системы, что выгодно отличает этот метод от волнодисперсного РФА. Поэтому ЭД-РФА-спектрометры компактны и надежны в эксплуатации.

Программное обеспечение AnalytiX спектрометра Genius IF позволяет автоматически создавать различные процедуры калибровки по стандартным образцам с известными концентрациями. Также в нем реализована возможность определения концентраций без стандартных образцов с помощью метода фундаментальных параметров, в котором расчет содержания элементов производится по встроенным теоретическим зависимостям [2].

В ЭД-РФА очень важным фактором является загрузка детектора. ЭД-детектор способен воспринять ограниченный поток квантов без потери линейности и разрешения, поэтому необходимо максимально уменьшать рассеянное излучение трубки, создающее сплошной фоновый сигнал. Обычно применяют специальные фильтры, которые размещаются на пути рентгеновского пучка, между рентгеновской трубкой и образцом (см. рис. 1). Фильтр поглощает часть излучения в определенном небольшом спектральном интервале, понизив фон там, где расположены пики интересующих нас элементов. Однако, в остальных участках спектра фон сохраняется, ухудшая отношение сигнал/ шум и загружая детектор. Использование набора вторичных мишеней позволяет значительно улучшить эффективность работы детектора, сохраняя низкий фоновый сигнал во всем диапазоне спектра [3, 4].

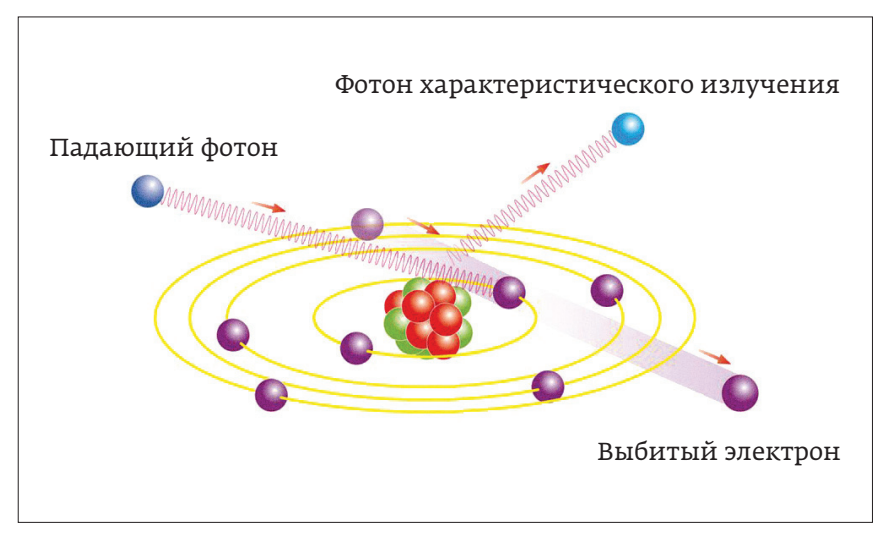

Puс. 2. Возникновение характеристического излучения

\section{ПРИНЦИП РАБОТЫ ВТОРИЧНЫХ МИШЕНЕЙ}

Вторичная мишень облучается источником и испускает свое собственное характеристическое излучение аналогично аноду в рентгеновской трубке. На рис. 3 видно, что мишень выступает в качестве источника, излучение от которого попадает на образец. Конечно, вторичная мишень рассеивает часть падающего на нее излучения, однако интенсивность его невелика, поскольку мишени изготавливают преимущественно из тяжелых материалов, в которых преобладает рэлеевское рассеяние. Необходимое условие работы со вторичными мишенями состоит в том, чтобы энергия рентгеновского излучения от вторичной мишени была выше энергии связи электронов в атомах образца, то есть энергии возбуждения интересующей нас характеристической линии.

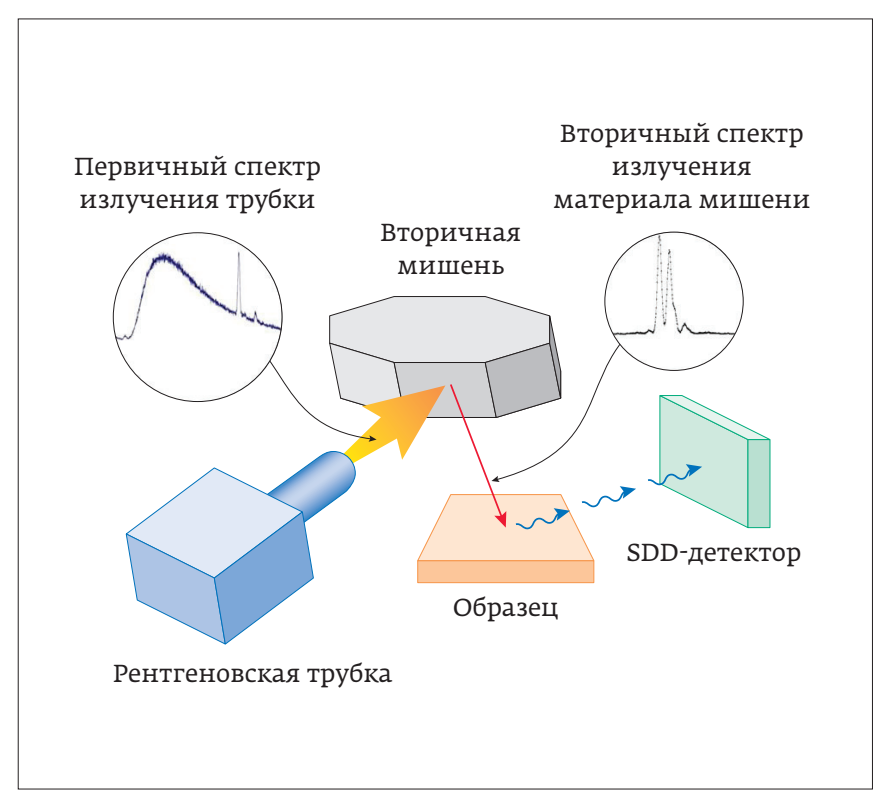

Puс. 3. Принцип работы вторичных мишеней 


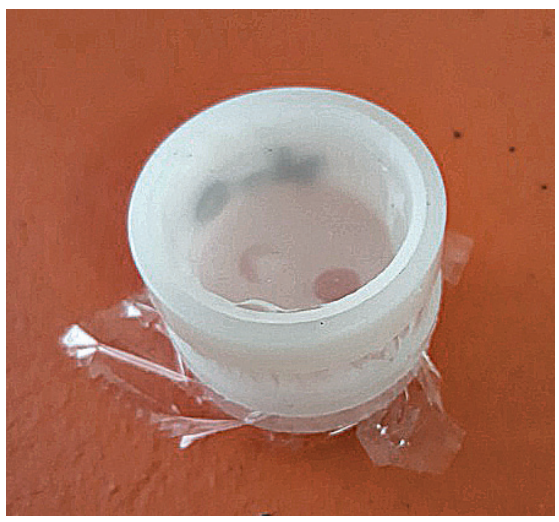

Puc. 4. Кювета

с образиом

\section{СРАВНЕНИЕ СПЕКТРОВ ИЗЛУЧЕНИЯ, ПОЛУЧЕННЫХ ПРИ РАЗНЫХ СПОСОБАХ ВОЗБУЖДЕНИЯ ОБРАЗЦА}

В качестве исследуемого образца использовали стандартный раствор 100 ppm Mn, Fe, Cu, Zn, As в 2\% $\mathrm{HNO}_{3}$. Образец помещали в кювету и накрывали защитной пленкой толщиной 4 мкм из ультралена (ultralene), которую фиксировали прижимным кольцом (рис. 4).

Все измерения проводили на ЭД-РФА-спектрометре Genius IF производства Xenemetrix (Израиль) (рис. 5а), в котором реализовано возбуждение образца с восьмью программно-управляемыми фильтрами и восьмью вторичными мишенями. Управление прибором происходит с помощью встроенного компьютера. Спектрометр оборудован рентгеновской трубкой мощностью 50 Вт и каруселью на восемь ячеек для образцов (рис. 5б), технические характеристики представлены в табл. 1.

Данные обрабатываются с помощью программного обеспечения Analytix (рис. 6) с автоматической идентификацией пиков элементов в получаемых спектрах и выводятся в меню в виде таблицы химических элементов с отображением оптимальных фильтров, которые рекомендуется использовать при исследовании содержания конкретных элементов в образце.

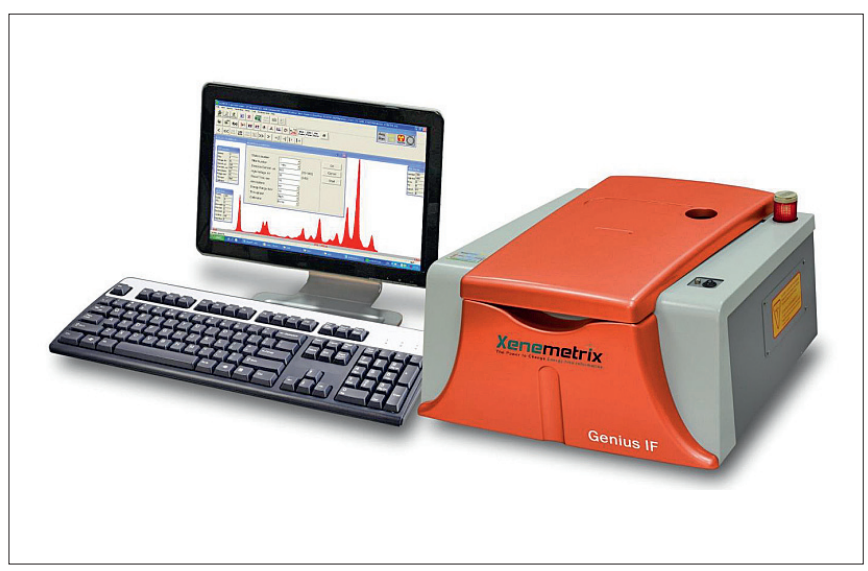

Puc. 5a. Cпектромеmp Genius IF таблица 1. Технологические характеристики исследовательского оборудования

\begin{tabular}{|c|c|}
\hline Наименование спектрометра & Genius IF \\
\hline $\begin{array}{l}\text { Диапазон обнаружения } \\
\text { элементов }\end{array}$ & $\mathrm{Na}(11)-\mathrm{U}(92)$ \\
\hline Диапазон концентраций & От долей ррт до 100\% \\
\hline Рентгеновские трубки & Rh/Ag/Mo/W/Pd-аноды \\
\hline Рентгеновская трубка & $50 \mathrm{kB}, 50 \mathrm{BT}$ \\
\hline Стабильность источника & $0,1 \%$ \\
\hline Тип окошка детектора & $\mathrm{Be}$ \\
\hline Карусель & 8 позиций \\
\hline Рабочая среда & Воздух/вакуум / гелий \\
\hline Количество фильтров & 8 \\
\hline Вторичные мишени & $\begin{array}{l}\mathrm{Si}, \mathrm{Ti}, \mathrm{Fe}, \mathrm{Zn}, \mathrm{Ge}, \mathrm{Zr}, \mathrm{Mo}, \mathrm{Sn} \\
\text { (стандартная } \\
\text { комплектация) }\end{array}$ \\
\hline Сменный коллиматор & $0,3-3,0 \mathrm{~mm}$ \\
\hline Питание & $110-230 \mathrm{~V}$ AC $50 / 60 \mathrm{~Hz}$ \\
\hline Размеры камеры & $22 \times 22$ см, глубина 5 см \\
\hline Программное обеспечение & $\begin{array}{l}\text { AnalytiX advanced package } \\
\text { (Microsoft Windows OS) }\end{array}$ \\
\hline Габаритные размеры & $55 \times 55 \times 32 \mathrm{CM}$ \\
\hline Bec & $50 \mathrm{kr}$ \\
\hline
\end{tabular}

\section{МЕТОДЫ ПОЛУЧЕНИЯ ХАРАКТЕРИСТИЧЕСКИХ СПЕКТРОВ ЭЛЕМЕНТОВ}

Прямое возбуждение образца

На рис. 7 представлен спектр, полученный в результате прямого возбуждения. Высокий фон делает подобный режим

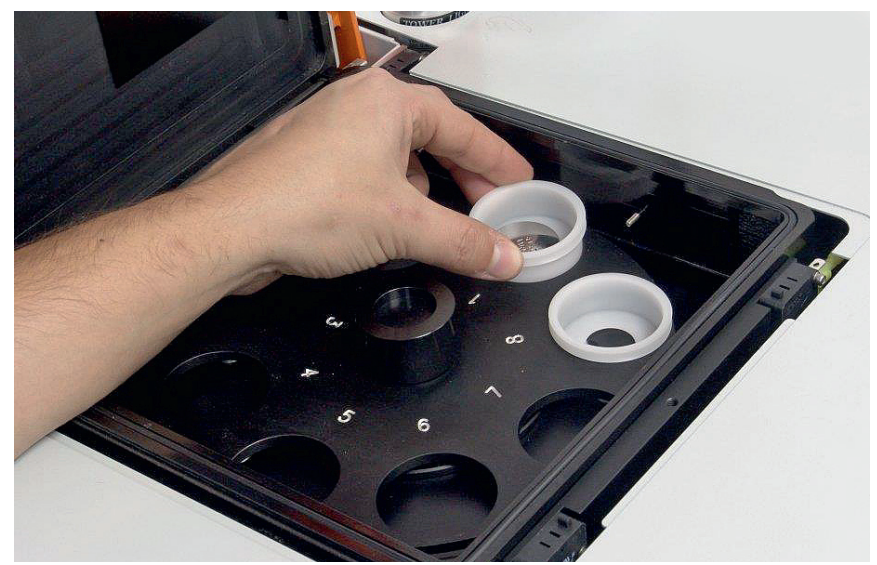

Puc. 56. Карусель для образщов 

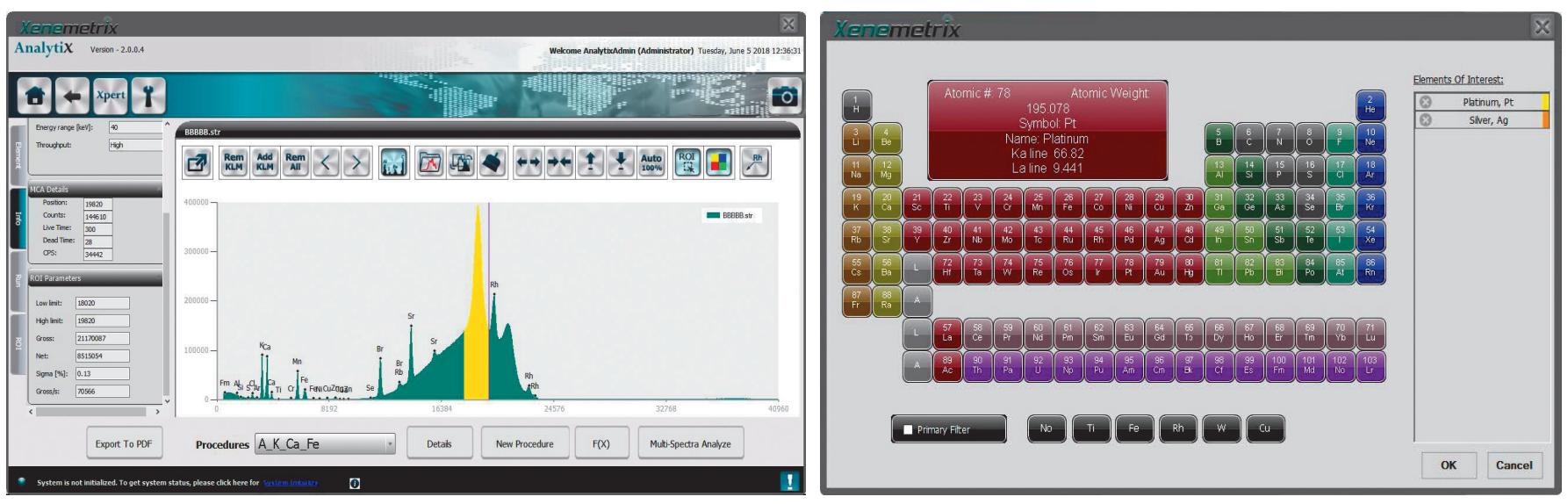

Puc. 6. Интерфейс программного обеспечения Analytix

съемки непригодным для определения малых концентраций многих элементов в образце. Такой высокий уровень шумов обусловлен рассеянием на образце излучения, приходящего от рентгеновской трубки.

Из-за высокого фонового сигнала этот режим хорошо подходит только в качестве обзорного для качественного определения интересующих элементов в образце. Низкое соотношение сигнал / шум ухудшает предел обнаружения, особенно для легких элементов.

\section{Съемка с использованием фильтра}

На рис. 8 представлен спектр, полученный при съемке с использованием железного фильтра. Фильтр вырезает из спектра люминесценции полосу поглощения материала фильтра (выделена желтым цветом) и понижает фон в этой зоне. В остальных же участках спектра фон сохраняется.

Этот режим съемки оптимален для элементов с атомными номерами в интервале $A=1,05-1,2$ от атомного номера материала фильтра. Недостаток метода - локальное понижение

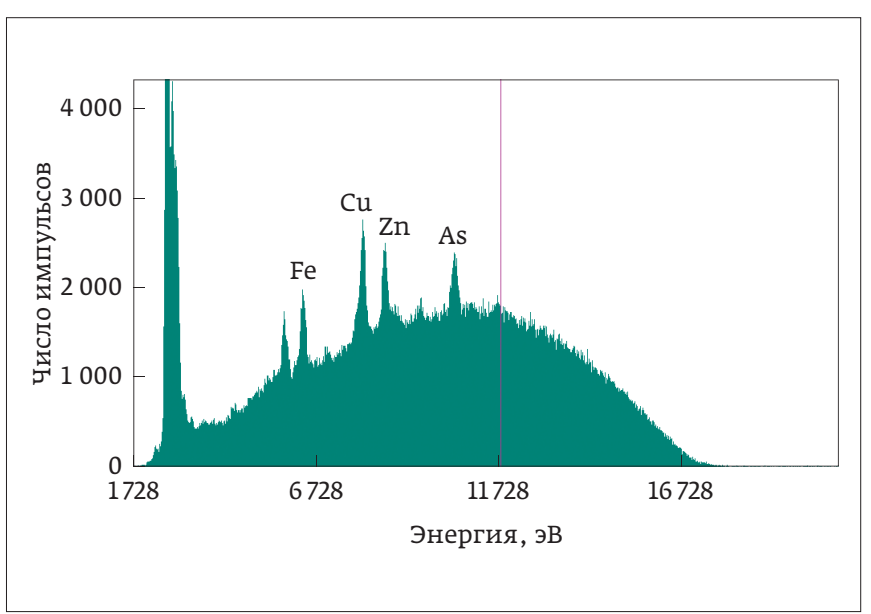

Puc. 7. Спектр, попученный в режиме прямого возбуждения фона в зоне поглощения материала фильтра, в то время как в других частях спектра фоновый сигнал остается прежним, а аналитические характеристики не улучшаются по сравнению с использованием прямого возбуждения.

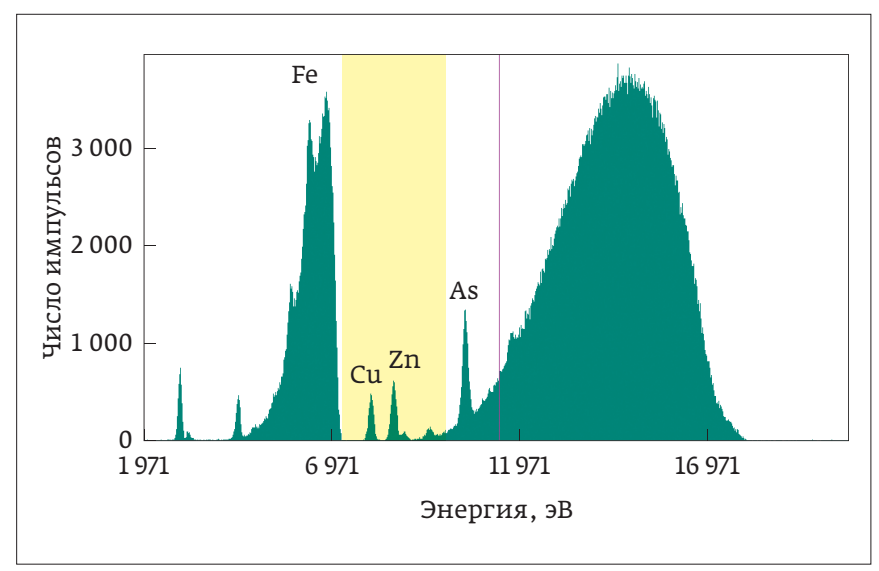

Puc. 8. Спектр, полученный с использованием Fе-фильтра

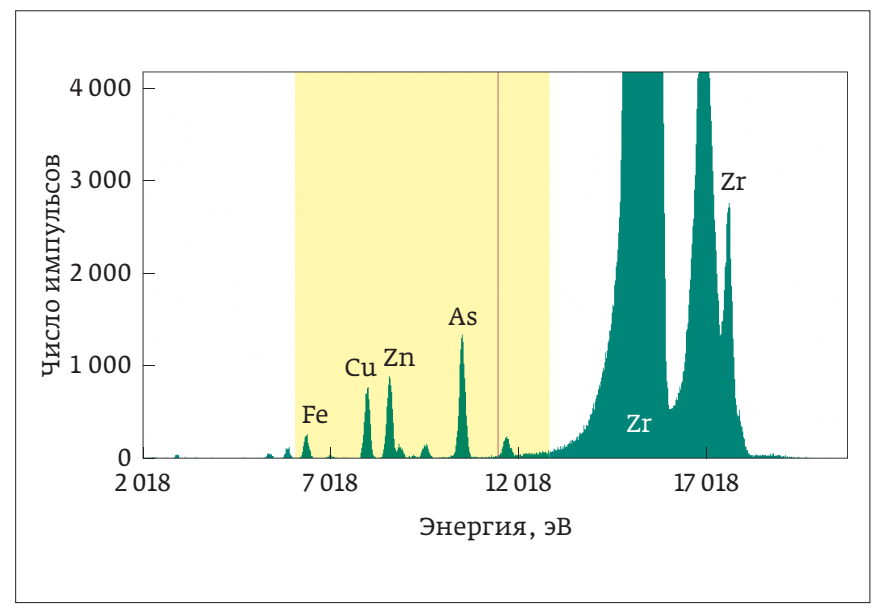

Puc. 9. Спектр, полученный с использованием Zr-вторичной мишени 


\section{Съемка с использованием вторичной мишени} На рис. 9 представлен спектр, полученный при съемке с циркониевой вторичной мишенью. Вторичная мишень переизлучает спектр трубки в виде узкой характеристической линии. Подобный вид энергетического спектра излучения позволяет обеспечить очень низкий фон практически во всем диапазоне, повышая чувствительность обнаружения бо́льшего числа элементов, чем при использовании фильтров (зона выделена желтым).

Способ оптимально подходит для съемки элементов с атомными номерами в интервале $A=0,5-0,95$ от атомного номера материала мишени. Использование вторичных мишеней при анализе обеспечивает очень низкий фон и, соответственно, высокое значение отношения сигнал / шум. Однако атомы с номером $A_{i} \geq A_{\text {мишени не будут воз- }}$ буждаться, поэтому необходим подбор материала мишени для обнаружения интересующих элементов в каждом конкретном измерении.

\section{Влияние материала мишени}

\section{на интенсивность линий аналитов}

Чтобы понизить предел обнаружения и повысить значение соотношения сигнал/шум, необходимо правильно подобрать материал мишени для конкретного измерения. На
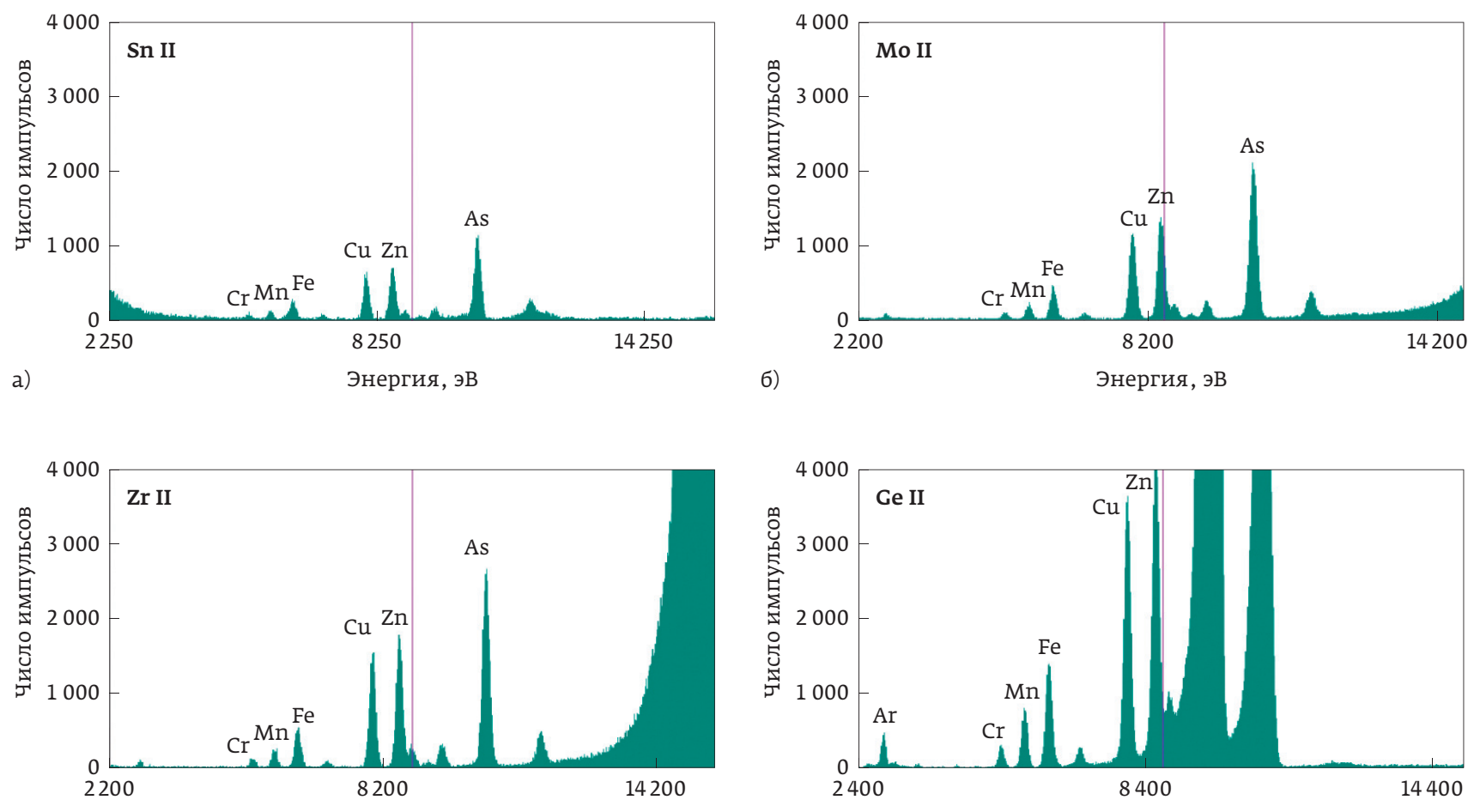

в)

Энергия, эВ
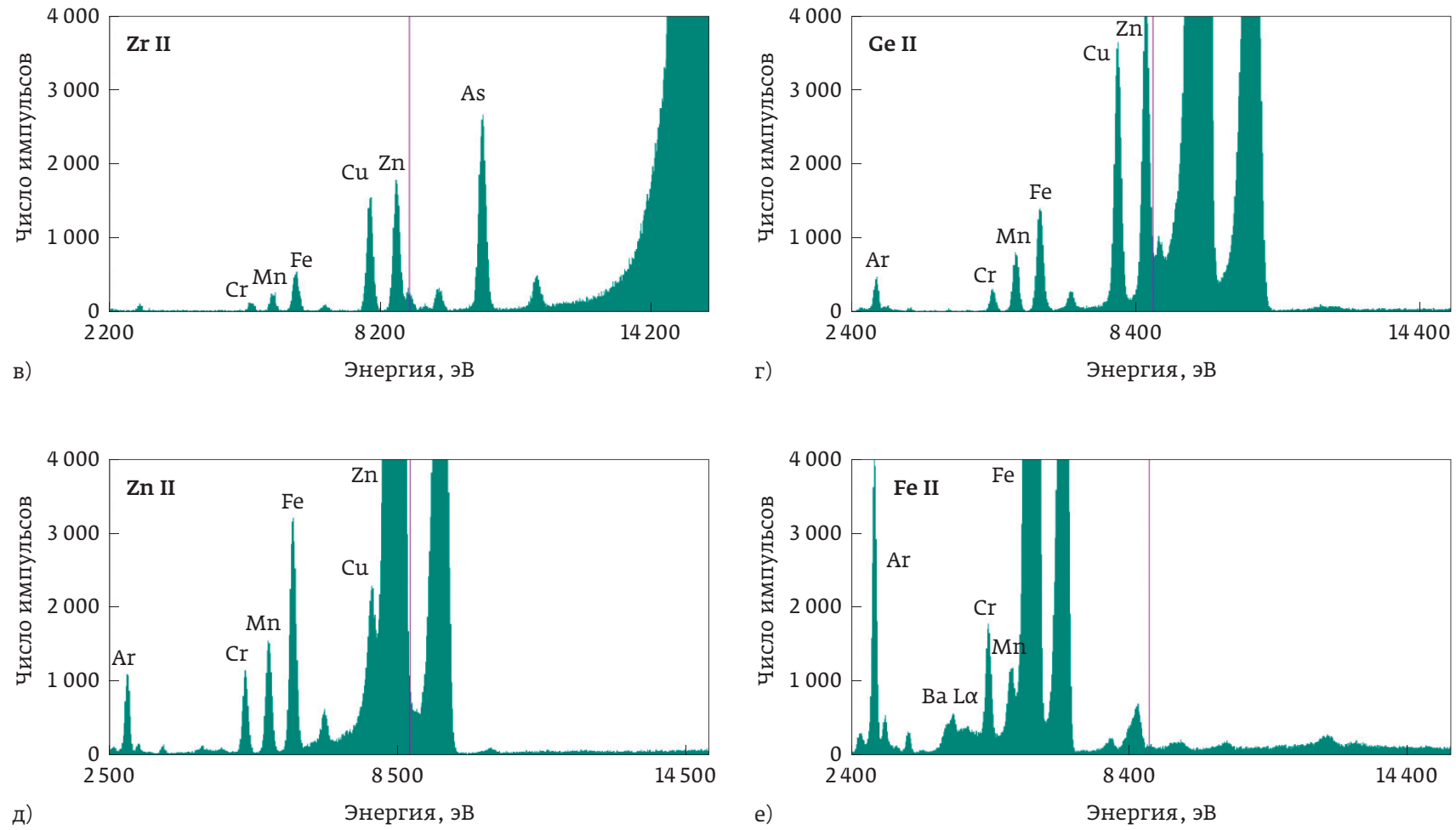

Puc. 10. а-спектр с Sn(50)-мишенью, 6-спектр с Мо(42)-мишенью, в - спектр с Zr(40)-мишенью, 2-спектр с Ge(32)-мишенью, д-спектр c Zn(30)-мищенью, е-спектр с Fe(26)-мищенью 
рис. 10а-е наглядно продемонстрировано влияние атомного номера материала мишени на вид получаемого спектра. Важно отметить, что правильный подбор мишени позволяет увидеть низкие концентрации некоторых элементов в образце, которые невозможно обнаружить при иных режимах съемки. В качестве исследуемого образца был выбран стандартный раствор 100 ppm As, Cd, Cu, Fe, $\mathrm{Mn}, \mathrm{Zn} \mathrm{в} 2 \% \mathrm{HNO}_{3}$.

На приведенных спектрах видно, что чем ниже атомный номер материала мишени, тем выше интенсивность линий для легких элементов. Применение железной мишени обеспечивает выраженный пик L-линии бария при концентрации 10 ppm при одновременной высокой чувствительности по легким элементам. Анализ полученных спектров еще раз доказывает необходимость вторичных мишеней для обнаружения низких концентраций легких элементов. В приборе также встроены мишени из Si(14) и Ті(22) для определения элементов, близких по атомной массе к $\mathrm{Na}$ - нижней границе диапазона чувствительности метода ЭД-РФА.

Обратим внимание, что при использовании мишени из материалов с атомным весом легче Zr (рис. 10в-е) появляется пик аргона, который, конечно, не содержится в образце. Его характеристическая линия происходит из находящегося в камере воздуха, который также способен к флуоресцентному излучению. Присутствие воздуха снижает чувствительность прибора в области легких элементов, однако эта проблема легко решается вакуумированием рабочей камеры или продувкой ее гелием. Эти функции уже встроены в спектрометр Genius IF, поэтому вместе со спектрометром предусмотрена поставка вакуумного насоса и/или гелиевых баллонов.

\section{Примеры спектров}

с использованием вторичных мишеней

Продемонстрируем преимущества использования вторичных мишеней на примерах реальных исследовательских образцов.

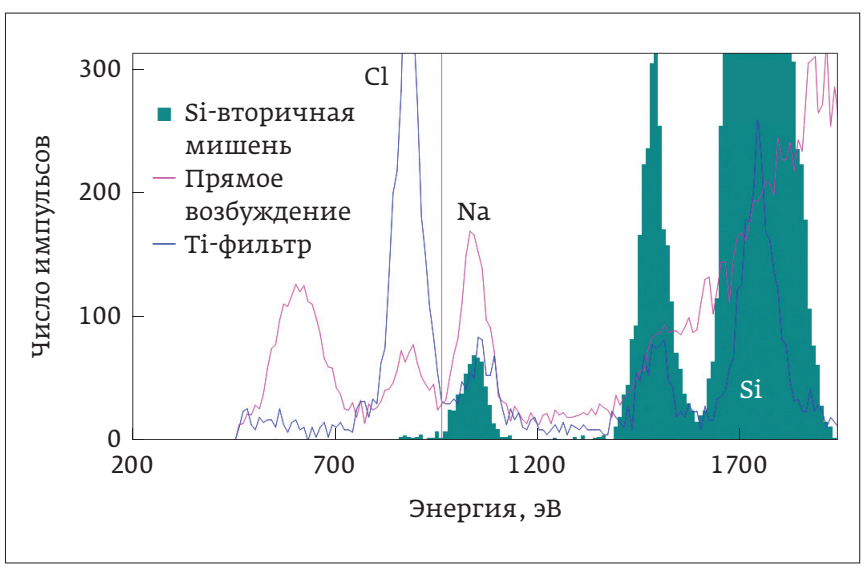

Puc. 11. Спектр смеси $\mathrm{H}_{3} \mathrm{BO}_{3} \mathrm{u} \mathrm{NaCl}$

\section{Образец: Смесь $\mathrm{H}_{3} \mathrm{BO}_{3}$ и $\mathrm{NaCl}$}

По рис. 11 видно, что использование кремния в качестве вторичной мишени позволяет значительно повысить соотношение сигнал / фон для легких элементов, в частности Na.

\section{Образец: $\mathrm{CuSO}_{4}$ в водном растворе, одноэлементная матрица}

На рис. 12 видно, что спектр образцов в водном растворе содержит рассеянное излучение трубки, что вызывает высокий уровень фона. Использование Мо-вторичной мишени позволяет устранить фон от рассеянного излучения во всем спектральном диапазоне.

\section{Образец: Латунь CDA443}

При исследовании образцов, содержащих элементы, чьи характеристические линии находятся очень близко, возникают проблемы в различении этих линий, так как часто они накладываются друг на друга. Спектр на рис. 13 показывает, что использование Sn-вторичной мишени позволило выделить пик As $K_{\beta}$, что дает возможность определять низкие содержания As в присутствии Рb.

\section{Образец: Геологические пробы}

На рис. 14 видно, что использование Мо-вторичной мишени позволяет охватить более широкий диапазон спектра и одновременно определять с высокой точностью большее количество элементов, чем использование фильтра, что может быть особенно важно при высокой рабочей загрузке прибора.

\section{вывод}

Использование вторичных мишеней для ЭД-РФА является необходимым условием для определения низких концентраций элементов в образцах. Переизлучение спектра рентгеновской трубки в виде узкой характеристической линии материала позволяет очень сильно понизить фоновый сигнал и добиться рекордных для данного метода

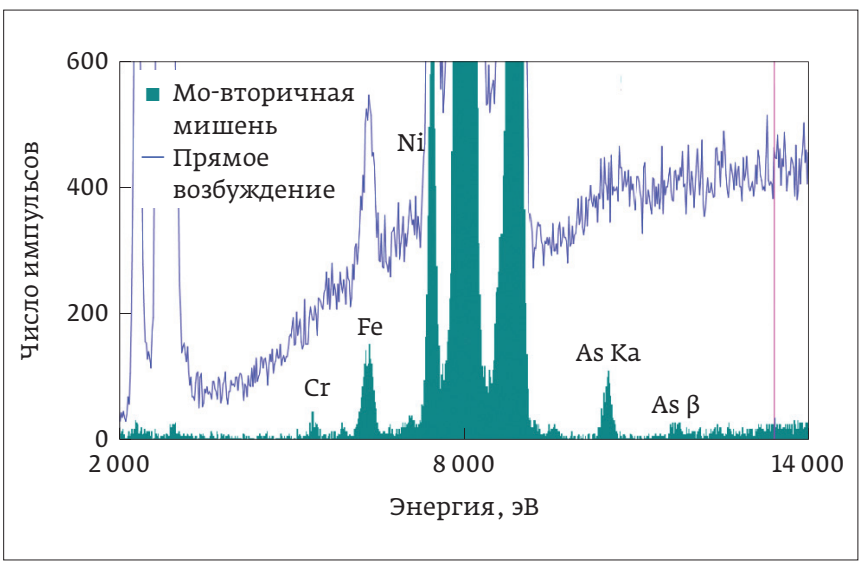

Puc. 12. Спекmр CuSO 4 в водном растворе 


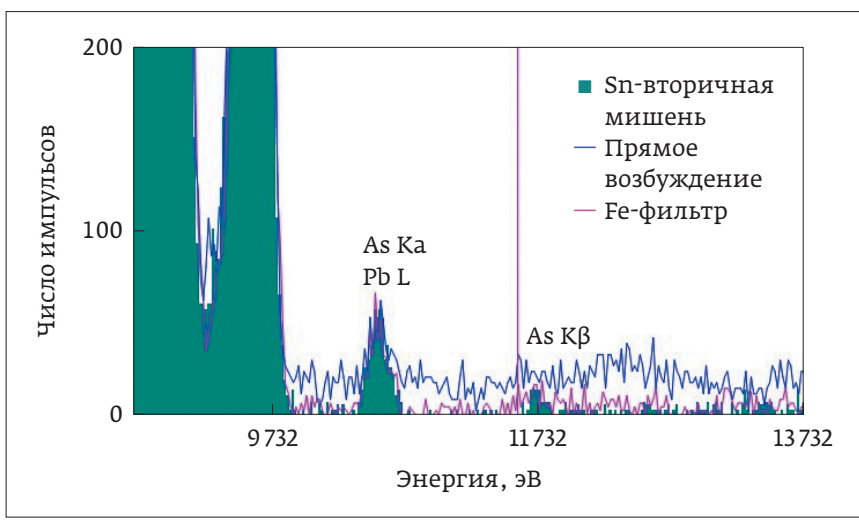

Puc. 13. Спекmр патуни CDA443

чувствительностей в широком диапазоне определяемых концентраций.

Применение вторичных мишеней расширяет пределы обнаружения метода без внедрения принципиальных усложнений конструкций ЭД-спектрометров, что является их большим преимуществом, как с точки зрения улучшения аналитических характеристик, так и с позиции экономической выгоды.

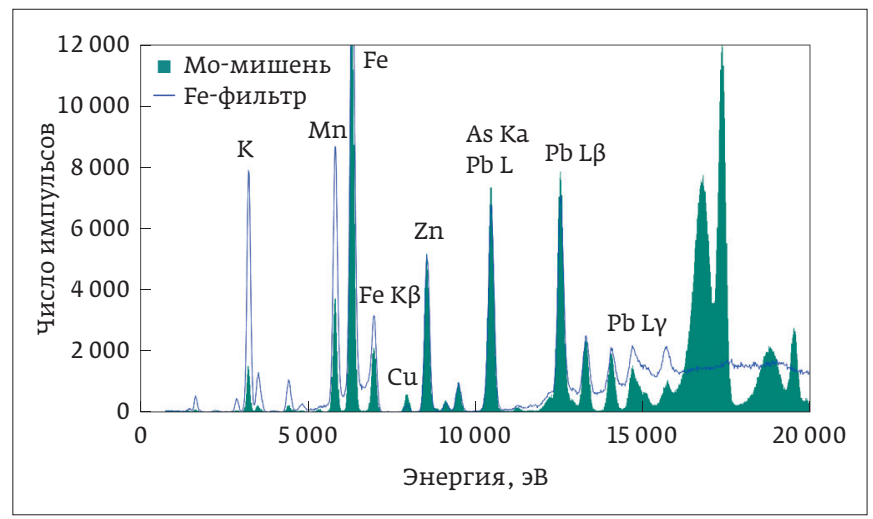

Puc. 14. Спектр геологического образиа

\section{ЛИТЕРАТУРА}

1. Embong Z., Wagiran H. Optimization of a Spectrometry for EnergyDispersive X-ray Fluorescence Analysis by X-ray Tube in Combination with Secondary Target for Multielements Determination of Sediment Samples / University Of Technology Malaysia. 2015. P. 111-118.

2. Bower P. Theory of XRF // PANanalytical BV. 2003. № 3. P. 1-62.

3. Dr. Rousseau R. Detection limit and estimate of uncertainty of analytical XRF results // The Rigaku Journal. 2001. V. 18. № 2. P. 33-47.

4. Jenkins R., Gilfrich J.V. Figures-of-merit, their philosophy, design and use // X-Ray Spectrometry. 1992. V. 21. № 6. P. 263-269.

\section{ИСЧЕЗАЮЩИЕ ПЛАСТИКИ СОХРАНЯЮТСЯ В ТЕНИ, НО ТАЮТ НА СОЛНЦЕ}

В мифе об Икаре крылья, тающие на солнце, привели к трагедии. А у военных США летающие аппараты, испаряющиеся под воздействием солнечного света, могут стать мощным оружием! Можно ли создать беспилотник, бесследно исчезающий после тайной миссии в отдаленном месте?

Инженеры Технологического института Джорджии разработали новый тип пластика, из которого можно формировать гибкие листы и прочные механические детали, исчезающие в течение нескольких минут или часов при воздействии УФ или температуры выше $80^{\circ} \mathrm{C}$. До сих пор попытки сделать самоуничтожающиеся пластмассы упирались в проблему предотвращения разрушения при комнатной температуре. Новое вещество, созданное командой Tech Ceorgia, может оставаться стабильным в течение многих лет, пока не подвергнется воздействию солнечного излучения. Эта пластмасса может использоваться для изготовления датчиков окружающей среды и медицинских приборов, растворяющихся после сбора данных, или временных клеев, отклеивающихся с помощью теплового пистолета. У Агентства перспективных исследовательских проектов в области обороны (DARPA) есть программа финансирования исследований по созданию исчезающих средств доставки, включая разработки Georgia Tech.

Пластик состоит из синтетических полимеров, свя занных сверхсильными связями. Возврат их к блокам требует разрушения каждой связи, подобно разрыву ожерелья. Поскольку на этот процесс влияют влажность, кислотность, температура и другие факторы окружающей среды, разрушение может занять месяцы. Существует много медленно разлагающихся пластиков Для создания прочных пластмасс, разрушающихся «по команде», обратились к молекуле полифталальдегида (РРНА). Как и все полимеры, он распадается на блоки при нагревании выше определенной температуры, но, в отличие от пластиков, используемых в бутылках и упаковке, РРНА распадается при температурах ниже комнатной. Чтобы сделать РРНА псевдостабильным, ученые связали цепь молекул в круг. Цепи, соединенные вместе, труднее разрываются. Хотя круговая структура предотвращает разрушение модифицированного РРНА при комнатной температуре, достаточно триггеру разорвать хоть одну связь, вещество мгновенно деполимеризуется и полностью разлагается.

Для разложения РРНА на солнце добавили соединение, которое становится кислым в присутствии солнечного света, а затем атакует связи РРНА. Для

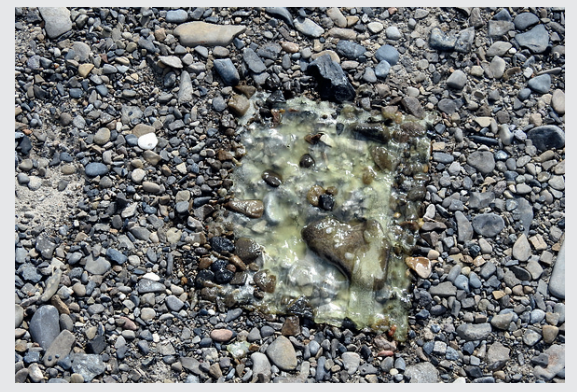

Puc. 1. Деградация попимера РРНА на сопнще

регулировки скорости деградации (предоставляя беспилотнику время для миссии) были добавлены соединения, реагирующие с кислотой и ослабляющие эффект ее воздействия. Удаление примесей, в основном трифторида бора, увеличивает срок годности материала до 20 лет при комнатной температуре в помещении.

Несмотря на замечательные свойства, пластик сложен в использовании из-за хрупкой текстуры. Авторы проекта утверждают, что эта проблема уже решена, и вскоре их результаты можно будет применить в промышленном производстве.

Scientific American 


\section{Московский международный}

Салон изобретений

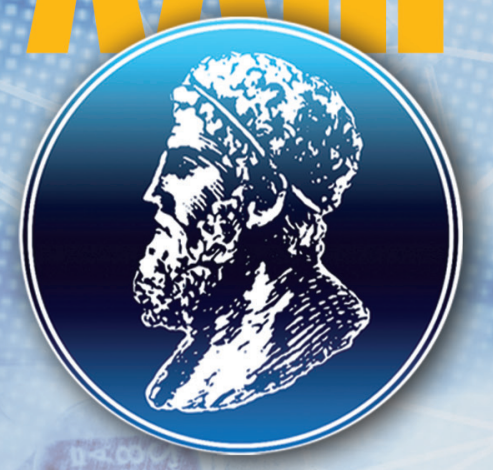

и инновационных технологий

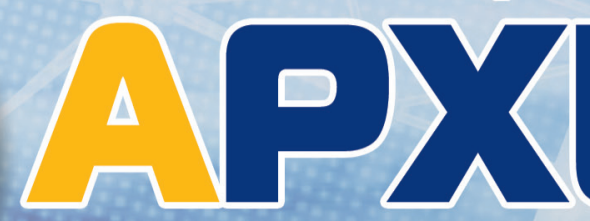

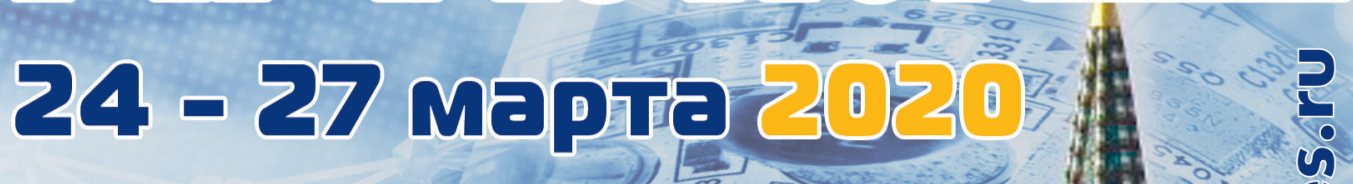

Москва, Россия, Конгрессно-выставочный центр "Сокольникй", поввйьон №2

Конкурсная программа

Презентация высокотехнологичных проектов

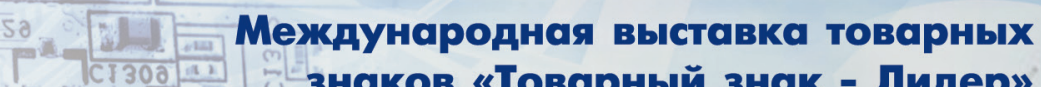
3наков «Товарный знак - Лидер»

Международная научно-практическая конференция «Актуальные вопросы изобретательской, и патентно-лицензионной деятельности»

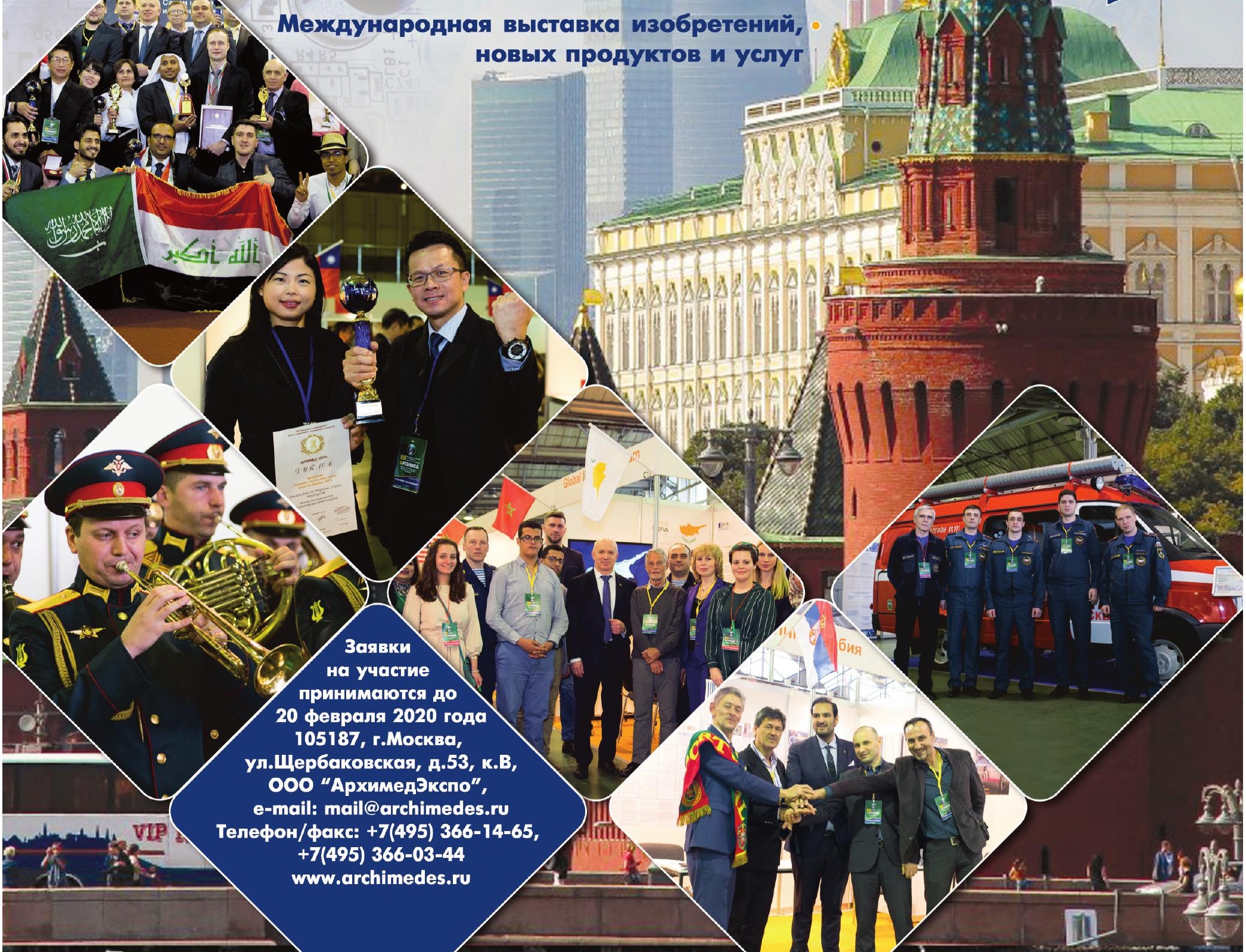

